\title{
TransRadial versus transUlnar artery approach for elective invasive percutaneous coronary interventions: a randomized trial on the feasibility and safety with ultrasonographic outcome - RAUL study
}

\author{
Dagmara Gralak-Lachowska ${ }^{1}$, Paweł J. Lewandowski ${ }^{1}$, Pawel Maciejewski ${ }^{1}$, Bogumił Ramotowski ${ }^{1}$, \\ Andrzej Budaj ${ }^{1}$, Sebastian Stec ${ }^{2}$ \\ ${ }^{1}$ Department of Cardiology, Centre of Postgraduate Medical Education, Grochowski Hospital, Warsaw, Poland \\ ${ }^{2}$ MediNice Research and Development Centre, Rzeszow/Krosno, Poland
}

Adv Interv Cardiol 2020; 16, 4 (62): 376-383

DOI: https://doi.org/10.5114/aic.2020.101761

\begin{abstract}
A bstract
Introduction: Transradial access (TRA) for coronary angiography (CAG) and percutaneous coronary intervention (PCI) is superior to transfemoral access (TFA). Transulnar access (TUA) is an alternative to TRA.

Aim: To compare the efficacy and safety of TRA vs. TUA in patients scheduled for CAG or PCI.

Material and methods: This was a prospective, single-center, randomized study conducted between 2013 and 2016. Two hundred patients referred for the first elective CAG were included in the study. Eligible patients were then randomly assigned to the TRA or TUA group. Before and after the invasive procedure, all patients underwent ultrasonographic measurements of the right upper limb arteries.

Results: The primary endpoint was efficacy, defined as a successful CAG without a crossover of vascular access. The secondary endpoint was safety, assessed as the number of vascular complications. Successful coronary angiography via the access site was $95 \%$ vs. $75 \%$ in the TRA vs. TUA groups, respectively $(p<0.001)$. It depended on the anatomy of UA and the operator experience. No differences were observed in early and late follow-up complications.

Conclusions: TRA was superior to TUA with regard to efficacy. TUA occurred a safe approach for CAG and PCI and could be used as an alternative method of forearm access.
\end{abstract}

Key words: transulnar access, complications, ultrasonography.

Su m m a ry

Direct comparisons of transradial access (TRA) and transulnar access (TUA) have shown different efficacy and feasibility outcomes. However, no clinical or ultrasonographic risk factors for unsuccessful access attempts, as well as long-term artery patency, have been validated. No clinical or ultrasonographic risk factors for unsuccessful access attempts, as well as longterm artery patency, have been validated for TUA. Thus, our study attempt to fill this gap in the literature. Our results reveal that TUA is a safe approach for coronary angiography and can be used as an alternative method of forearm access. Operators may routinely use this approach to maintain their skills after an early learning curve to achieve a high rate of ulnar artery cannulation.

\section{Introduction}

The diagnosis of coronary artery disease and invasive treatment requires vascular access. The radial artery approach for coronary angiography (CAG) and percutaneous coronary intervention $(\mathrm{PCI})$ is superior to the transfemoral access (TFA) in patients with acute coronary syndromes and patients scheduled for elective procedures [1-3]. Accordingly, large-scale randomized controlled trials and registries showed decreased incidence of major bleeding and vascular complications as well as

\section{Corresponding author:}

Paweł J. Lewandowski MD, PhD, Department of Cardiology, Centre of Postgraduate Medical Education, Grochowski Hospital, Warsaw, Poland, phone: +480501352818, e-mail: levis@vita-medica.pl

Received: 9.06.2020, accepted: 22.08.2020. 
lower all-cause mortality in patients allocated to transradial access (TRA) compared with TFA [4]. However, in up to $10 \%$ of attempted cases for CAG, TRA may not be successful. Transulnar access (TUA) was originally proposed as a viable alternative forearm approach in patients who are unable to undergo TFA $[5,6]$. The ulnar artery (UA) has a larger diameter and straighter anatomical pattern and is less prone to spasm, which may facilitate easier cannulation. Direct comparison of TRA and TUA showed different efficacy and feasibility results [7-9].

No clinical or ultrasonographic risk factors for unsuccessful access attempts, as well as long-term artery patency, have been validated.

\section{Aim}

The aim of this study was to compare TRA and TUA in patients scheduled for elective CAG with regard to efficacy and safety.

\section{Material and methods}

This was a prospective, single-center, randomized study conducted between 2013 and 2016. The first patient was randomized in September 2013 and the 6-month follow-up was completed in September 2016. Two hundred patients referred for the first elective CAG were included in the study. Eligible patients were then randomly assigned to the TRA or TUA group. Randomization was performed using the sealed envelope method. The ethics review board of the Centre of Postgraduate Medical Education approved the study. Informed consent was obtained from all the participants. The study was performed in accordance with the Declaration of Helsinki.

CAG was performed by experienced certified interventional cardiologists. One operator (operator 1) was an expert in both TRA and TUA procedures (more than 100 procedures with TUA), the second (operator 2) was an expert in TRA and had intermediate TUA expertise (between 50 and 100 procedures with TUA), while the third (operator 3) was an expert in TRA and a novice in TUA.

Regardless of sex, patients who were at least 18 years of age and hospitalized for their first elective CAG were eligible for the trial. Patients with upper limb anomalies and those who underwent previous vascular interventions (TRA or TUA) were excluded. Additional exclusion criteria were as follows: patients with RA or UA that were less than $1.5 \mathrm{~mm}$ in diameter (based on pre-procedural ultrasound examination) and patients with positive Allen's test results. Only 4 persons were excluded from the study based on the ultrasonographic criteria.

Before the invasive procedure, all patients underwent two-dimensional ultrasonography and Doppler measurements of the right upper limb arteries. Ultrasonography was performed by one investigator. The vessel diameter, distance from the skin to the anterior wall of an artery (depth of the artery), as well as the peak systolic velocity of the blood in the radial artery (RA) and UA were measured. All parameters were assessed $2 \mathrm{~cm}$ above the wrist at the site of potential puncture. The interventional cardiologists were blinded to the ultrasound outcomes. Follow-up ultrasound examinations were performed $24 \mathrm{~h}, 1$ month, 3 months and 6 months after CAG/PCI. There was no standard protocol with saline hydration or using tranquilizers before CAG was performed - those were provided individually for every patient.

The primary endpoint was efficacy, defined as a successful CAG without crossover of vascular access (successful coronary angiography via the randomized access site).

The secondary endpoint was safety, assessed as the combined number of early vascular complications (total artery occlusion (TAO), pseudoaneurysm, large hematoma of the forearm (grade 4 in EASY scale)) [10]. Follow-up ultrasonography was performed and data on long-term TAOs (after 30 days, 3 and 6 months) were also analyzed.

The visual analog scale (VAS) was applied for the evaluation of patient comfort associated with vascular access. Right upper limb access was the first choice. Standard 6-Fr radial introducers (Radial Introducer Sheath, Demax Medical Co. Ltd., Sydney, Australia), 6 or 5-Fr diagnostic catheters (Angiodyn, B Braun, Melsungen, Germany), and 6- Fr guiding catheters (Launcher, Medtronic, Danvers, USA) were used for CAG. If the primary access was unsuccessful, the next site of vascular access was based on the physician's decision (crossover to the ipsilateral vessel or switch to the contralateral RA or UA). After vascular access was established, a bolus of 5000 IU of unfractionated heparin and $0.2 \mathrm{mg}$ of intra-arterial nitroglycerine was administered to prevent vascular spasm. Further, angiography of the upper limb circulation was recorded. In the case of $\mathrm{PCl}$, the heparin dose was 70-100 IU/kg. Several procedural parameters were prospectively assessed, including vascular access achievement time (time between first attempt of puncture and vascular sheath placement), fluoroscopy time, radiation dose and volume of the contrast. According to standard protocols, CAG was performed along with $\mathrm{PCl}$ ad hoc if necessary. Upper limb angiography was performed to evaluate circulation before the removal of the vascular sheath after CAG/PCI. Angiography of forearm arteries was also performed when there were difficulties with introducing the catheter. Before CAG consented subjects were randomized to one of two hemostasis protocols: QuikClot (QC) - a hemostatic device that is composed of a kaolin-impregnated sterile roll and system of direct wound pressure, or standard radial artery gauze compression (SC) - both with short manual compression at the beginning and continuous pressure for at least $2 \mathrm{~h}$ afterward. A comparison of the two methods was presented in a separate article [11].

\section{Statistical analysis}

The safety endpoint outcomes were assessed on an on-treatment analysis, which included all patients with 
completed $\mathrm{CAG} / \mathrm{PCI}$. The analysis of efficacy was examined on an intention-to-treat basis in all patients who underwent randomization and assigned to TRA or TUA. The distribution of quantitative variables is presented as mean \pm SD or median and interquartile range if deviation from the normal assumption was observed using the Shapiro-Wilk test.

Differences in these variables were compared using Student's $t$-test or the Mann-Whitney U-test as appropriate. Qualitative variables are summarized as numbers and percentages and were compared using the $\chi^{2}$ test or Fisher's exact test when the number of expected events was less than 5. A two-sided $p$-value of equal or less than 0.5 was considered statistically significant. Multiple logistic regression models were fitted to identify independent predictors for binary endpoints at a $5 \%$ level of statistical significance. Odds ratios (OR) with 95\% confidence intervals $(\mathrm{Cl})$ were provided for significant predictors. The following factors were analyzed: age, male gender, body mass index (BMI), diameter of UA, distance from the skin to UA and experience of operators. SAS software version 9.2 (SAS Institute, Cary, NC) was used in all the analyses.

\section{Results}

Between 2013 and 2016, 200 patients were randomized in a $2 \times 2$ factorial design between: right TRA versus TUA, and QC vs. SC at the vascular access site. The main indication for CAG was a suspected coronary artery disease. Baseline characteristics of the patients are summarized in Table I. No significant differences were observed between the TRA and TUA groups. Among 200 patients, $13(6.5 \%)$ were lost to long-term follow-up. Therefore, 187 patients were included in the analysis of the secondary endpoint, of which 94 and 93 were assigned to the TRA and TUA groups, respectively. These patients had evaluable data for 6-month follow-up.

Before CAG, acetylsalicylate and clopidogrel were routinely administered to the patients in the preparation for

Table I. Demographic and baseline characteristics of the patients and concomitant medications

\begin{tabular}{|c|c|c|c|}
\hline Parameter & $\begin{array}{c}\text { TRA } \\
n=100\end{array}$ & $\begin{array}{c}\text { TUA } \\
n=100\end{array}$ & $P$-value \\
\hline Age, mean \pm SD [years] & $66.0 \pm 8.8$ & $66.3 \pm 10.7$ & 0.88 \\
\hline Sex, male, $n(\%)$ & $41(41)$ & $52(52)$ & 0.12 \\
\hline $\mathrm{BMI}$, mean $\pm \mathrm{SD}\left[\mathrm{kg} / \mathrm{m}^{2}\right]$ & $29.4 \pm 4.1$ & $28.6 \pm 5.5$ & 0.29 \\
\hline Smokers, $n(\%)$ & $17(17)$ & $13(13)$ & 0.43 \\
\hline Hypertension, $n(\%)$ & $81(81)$ & $81(81)$ & 1.00 \\
\hline Hypercholesterolemia, $n$ (\%) & $70(70)$ & $77(77)$ & 0.26 \\
\hline Peripheral artery disease, $n(\%)$ & $9(9)$ & $10(10)$ & 0.80 \\
\hline Diabetes, $n(\%)$ & $37(37)$ & $25(25)$ & 0.06 \\
\hline Stroke, $n(\%)$ & $6(6)$ & $9(9)$ & 1.00 \\
\hline Renal insufficiency, $n$ (\%) & $5(5)$ & $2(2)$ & 0.44 \\
\hline Suspected CAD, $n(\%)$ & $97(97)$ & $98(98)$ & NA \\
\hline Heart valve disease, $n(\%)$ & $3(3)$ & $2(2)$ & NA \\
\hline Warfarin, $n(\%)$ & $7(7)$ & $7(7)$ & 1.00 \\
\hline New oral anti-coagulant, $n(\%)$ & $6(6)$ & $3(3)$ & 0.45 \\
\hline Statin, $n(\%)$ & $57(57)$ & $67(67)$ & 0.14 \\
\hline$\beta$-Blocker, $n(\%)$ & $75(75)$ & $77(77)$ & 0.74 \\
\hline Ca blocker, $n(\%)$ & $27(27)$ & $33(33)$ & 0.35 \\
\hline Aspirin, $n(\%)$ & 99 (99) & $98(98)$ & 0.5 \\
\hline Clopidogrel, $n$ (\%) & $97(97)$ & $97(97)$ & 1.00 \\
\hline Nitroglycerine i.a., $n$ (\%) & $100(100)$ & $100(100)$ & 1.0 \\
\hline Heparin, bolus of 5000 (IU) i.v., n (\%) & $100(100)$ & $100(100)$ & 1.0 \\
\hline Heparin, mean \pm SD [IU] & $6180 \pm 1788$ & $5965 \pm 1570$ & 0.36 \\
\hline
\end{tabular}

$B M I$ - body mass index, CAD - coronary artery disease, TRA - transradial access, TUA - transulnar access. 
Table II. Pre-procedural ultrasonographic data of the radial and ulnar artery and coronary angiography periprocedural data

\begin{tabular}{lccc} 
Parameter & TRA & TUA & P-value \\
& $n=100$ & $n=100$ & 0.58 \\
\hline Diameter of the artery, mean \pm SD $[\mathrm{mm}]$ & $2.47 \pm 0.35$ & $2.44 \pm 0.39$ & 0.01 \\
\hline Depth of the artery, mean \pm SD $[\mathrm{mm}]$ & $5.96 \pm 2.13$ & $6.73 \pm 2.30$ & 0.80 \\
\hline PSV1, mean \pm SD $[\mathrm{m} / \mathrm{s}]$ & $0.56 \pm 0.18$ & $64(64)$ & 0.88 \\
\hline No. of angiography alone, $n(\%)$ & $63(63)$ & $36(36)$ & 0.88 \\
\hline No. of PCl ad hoc, $n(\%)$ & $37(37)$ & $163.7 \pm 26.2$ & $<0.001$ \\
\hline Vascular access achievement time, mean \pm SD $[\mathrm{s}]$ & $58.8 \pm 45.3$ & $2.5(1.8-6.2)$ & 0.55 \\
\hline Fluoroscopy time $[$ min] & $2.95(1.4-5.6)$ & $243.0(144.5-474.5)$ & 0.25 \\
\hline Radiation dose of X-ray [mSv] & $303.0(145.0-486.3)$ & $117.0 \pm 62.5$ & 0.65 \\
\hline Contrast medium, mean \pm SD $[\mathrm{ml}]$ & $128.2 \pm 75.1$ & $50(50)$ & 1.0 \\
\hline QuikClot hemostatic device & $50(50)$ & $50(50)$ & 1.0
\end{tabular}

${ }^{1}$ Peak systolic velocity, TRA - transradial access, TUA - transulnar access.

$\mathrm{PCl}$. There was no statistically significant difference in the mean dose of heparin between the two groups ( $\mathrm{Ta}$ ble I). Diagnostic CAG was performed in all the patients, and ad hoc $\mathrm{PCl}$ was conducted in 37 (37\%) and 36 (36\%) patients in the TRA and TUA groups, respectively. Preprocedural ultrasonographic characteristics of the patients are summarized in Table II. UA was located more deeply than RA $(p=0.01)$. Concerning artery diameter and peak systolic velocity, no significant differences were observed between the groups. Angiographic and procedural data were similar in both groups, except for vascular access achievement time $(p<0.001)$ (Table II).

\section{Efficacy}

The angiographic success of vascular access was achieved in 95 (95\%) vs. 75 (75\%) in the TRA vs. TUA groups, respectively $(p<0.001)$ (Table III). In the TRA and TUA groups, crossover was required in $5(5 \%)$ cases and $25(25 \%)$ cases, respectively $(p<0.0001)$. In the TRA group, the artery was not punctured successfully in $2(2 \%)$ cases, and in 1 (1\%) case, the introduction of the vascular sheath was not successful, although the vessel was punctured successfully. In the remaining 2 cases in the TRA group, crossover was necessary because of the difficulty in continuing the procedure after a successful introduction of the vascular sheath. In 16 (16\%) cases in the TUA group, the artery was not punctured successfully, and in another 8 (8\%) cases, the vascular sheath was not successfully introduced. Moreover, crossover was necessary after the insertion of the diagnostic catheter in 1 case. Because of crossover events between the two groups, ultimately, TRA was used in 120 (60\%), TUA in $77(38 \%)$, and the brachial artery in $3(2 \%)$ patients. The main reasons for the unsuccessful procedures in the TRA or TUA approach are presented in Table IV. Vessel spasm, impalpable pulse, prolonged procedure and painful procedure were revealed more frequently in TUA. All patients had angiography of forearm vessels, and there was only one case of severe spasm in the TRA group visualized in angiography (reported in Table IV). All other difficulties (lack of pulse, impossibility of puncture of the artery) with TRA and TUA were probably due to vascular spasm, but we were not able to visualize it. The crossover frequency in the TUA group depended on the operator experience: operator $1-9.4 \%$, operator $2-9.5 \%$, and op-

Table III. Efficacy of the TRA and TUA

\begin{tabular}{|c|c|c|c|}
\hline Parameter & $\begin{array}{c}\text { TRA } \\
n=100\end{array}$ & $\begin{array}{c}\text { TUA } \\
n=100\end{array}$ & $P$-value \\
\hline CAG success, $n(\%)$ & $95(95)$ & $75(75)$ & $<0.0001$ \\
\hline Necessity of cross-over, $n(\%)$ & $5(5)$ & $25(25)$ & $<0.0001$ \\
\hline Successful vessel puncture, $n(\%)$ & $98(98)$ & $84(84)$ & 0.0005 \\
\hline Successful introduction of the vascular sheath, $n(\%)$ & $97(97)$ & $76(76)$ & $<0.0001$ \\
\hline
\end{tabular}

CAG - coronary angiography, TRA - transradial access, TUA - transulnar access. 
Table IV. Primary reasons for crossover in the TRA and TUA groups

\begin{tabular}{lccc} 
Parameter & TRA & TUA & $P$-value \\
& $n=100$ & $11(11)$ & 0.01 \\
\hline Vessel spasm, $n(\%)$ & $1(1)$ & $10(10)$ & 0.04 \\
\hline Impalpable pulse, $n(\%)$ & $3(3)$ & $10(10)$ & 0.04 \\
\hline Prolonged procedure, $n(\%)$ & $3(3)$ & $6(6)$ & 0.03 \\
\hline Painful procedure, $n(\%)$ & $0(0)$ & $2(2)$ & 0.50
\end{tabular}

TRA - transradial access, TUA - transulnar access.

Table V. Early and late clinical and ultrasonographic complication rates in TRA vs. TUA after crossover (on-treatment analysis)

\begin{tabular}{|c|c|c|c|}
\hline Complication & $\begin{array}{c}\text { TRA } \\
n=120\end{array}$ & $\begin{array}{c}\text { TUA } \\
n=77\end{array}$ & $P$-value \\
\hline TAO at $12 h, n(\%)$ & $2(1.7)$ & $1(1.3)$ & 1.00 \\
\hline Pseudoaneurysms at 12 h, $n$ (\%) & $2(1.6)$ & $2(2.5)$ & 1.00 \\
\hline Large hematoma at $12 \mathrm{~h}, n$ (\%) & $3(2.5)$ & $4(5.2)$ & 0.43 \\
\hline \multirow[t]{2}{*}{ Combined early local complications, $n$ (\%) } & $7(5.8)$ & $7(9)$ & 0.38 \\
\hline & $n=110$ & $n=74$ & \\
\hline TAO after 12 h-30 days, $n$ (\%) & 0 & 1 & \\
\hline TAO 30 day-3 months, $n(\%)$ & 0 & 2 & \\
\hline TAO 3-6 months, $n$ (\%) & 1 & 0 & \\
\hline Total number of TAOs at 6 months, $n(\%)$ & $3(2.7)$ & $4(5.4)$ & 0.35 \\
\hline
\end{tabular}

TAO - total artery occlusion, TRA - transradial access, TUA - transulnar access.

erator $3-24.7 \%$. In the multivariate regression analysis, the independent factors for CAG success using the TUA were: diameter of UA: $O R=12.54 ; 95 \% \mathrm{Cl}: 2.09-75.27$ $(p<0.005)$; depth of $\mathrm{UA}: \mathrm{OR}=1.33 ; 95 \% \mathrm{Cl}: 1.025-1.731$ $(p<0.03)$; operator 1 vs. $3 \mathrm{OR}=10.73$; $95 \% \mathrm{Cl}: 2.63-$ $43.75(p<0.009)$.

\section{Safety}

The secondary safety endpoint outcomes were assessed in an on-treatment analysis. No differences were observed in terms of the incidence of early and late complications between the groups. The frequency of combined vascular complications (TAO, pseudoaneurysms, and large hematoma) was similar in the TRA vs. TUA group (5.8\% vs. $7.7 \% ; p=0.38)$ in early observation. At 6-month follow-up there were 3 (2.7\%) vs. 4 (5.4\%) TAOs in the TRA vs. TUA group (Table V).

\section{Comfort treatment based on VAS}

Based on the analysis of VAS data, no statistically significant difference was observed in terms of treatment comfort depending on the type of vascular access 3.73 \pm 2.55 in TRA vs. $3.98 \pm 2.84$ in TUA; $p=0.24$.

\section{Discussion}

This single-center study demonstrates that TUA is a feasible and safe route for CAG. However, the efficacy of TUA was significantly lower compared to that of TRA. TUA could be used as a second-line approach when ipsilateral TRA is unattainable (the pulse over RA is weak) or should be preserved (coronary artery bypass grafting, arteriovenous fistula for hemodialysis). A meta-analysis by Dahal et al. compared the success rates, efficacy, and safety of TRA and TUA [12]. Data from five randomized clinical trials of 2744 patients were included in this analysis, with nearly an equal number of patients undergoing TRA- and TUA-guided catheterizations (1360 and 1384, respectively) $[6,9,10,13,14]$. The primary outcomes were major adverse cardiac events (MACE), and the secondary outcome was the composite endpoint of access-related complications. The authors concluded that TUA resulted in higher rates of access site failure and crossover. However, it had similar efficacy (similar MACE rates), safety (similar access site complications), and procedural times as TRA. Fernandez et al., who conducted a meta-analysis of six randomized controlled trials, also supported the use of UA as an alternative to RA for cardiac catheteriza- 

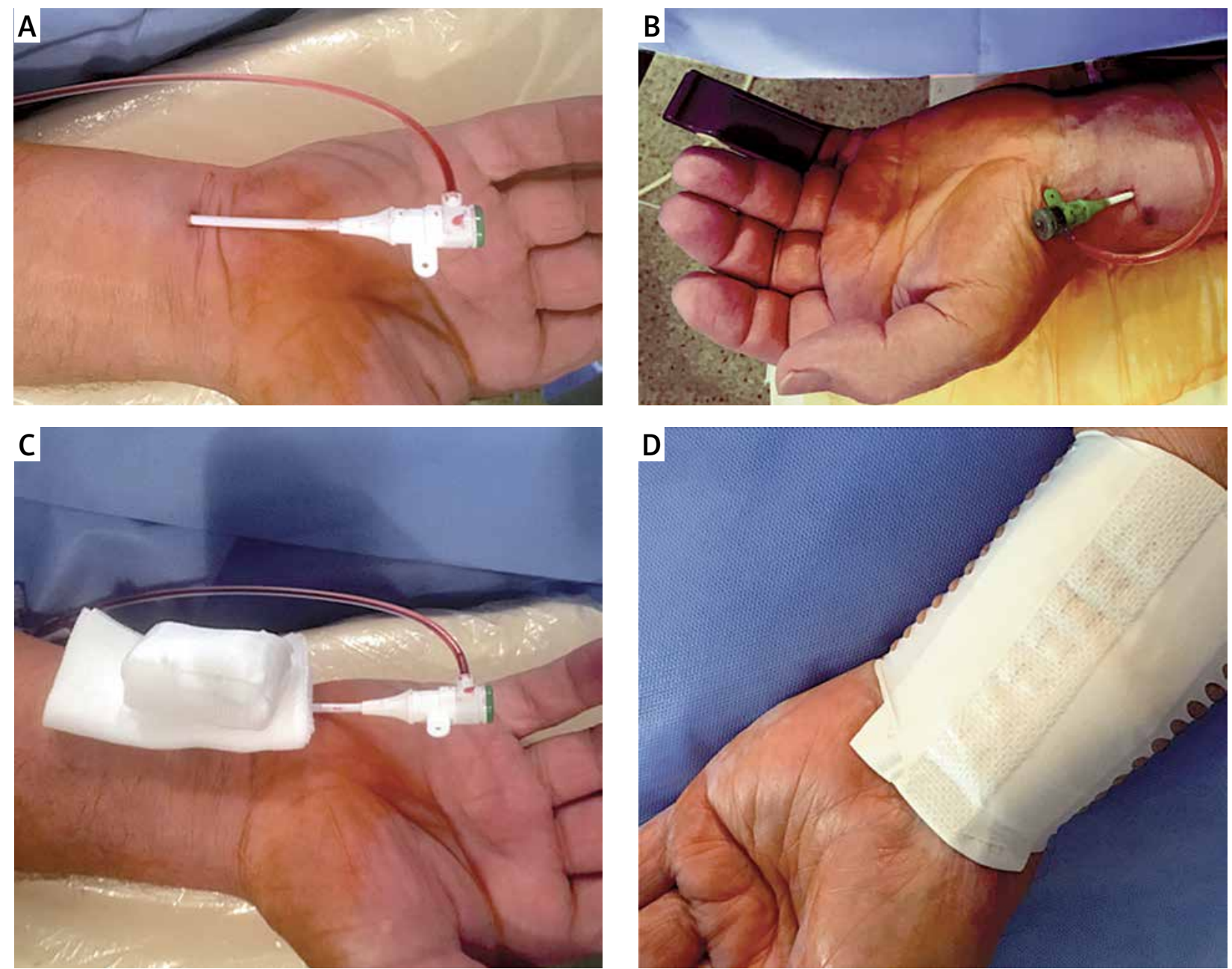

Figure 1. TRA and TUA access and two types of hemostasis - standard compression and QuikClot. A - TUA, B - TRA, C - standard compression in TUA, D - QuikClot compression in TRA

tion [15]. TUA is associated with fewer vascular complications, shorter time of hospitalization, and less patient discomfort [15-17]. TRA is not superior to TUA when the CAG/PCl is performed by an experienced operator [7]. Geng et al. reported, in 535 consecutive patients randomly assigned to TRA or TUA, that a successful puncture of the target artery was achieved in $95.1 \%$ and $91.5 \%$ of the patients in the TRA and TUA groups, respectively $(p>0.05)$ [14]. The utilization of UA as an access site increases the chance of success with forearm access and reduces the need for crossover to the femoral approach. The learning curve of this approach after appropriate guidance could be fast and safe. TUA can be difficult for the inexperienced operators because UA is generally less palpable than RA. UA is located more deeply, and the presence of several muscle tendons in the ulnar side of the wrist makes this artery more difficult to puncture. One of the largest trials (AURA of ARTEMIS), which tested the non-inferiority of TUA to TRA, was prematurely terminated because of the high crossover rates in TUA compared to TRA [9]. Anatomy reports suggest that UA is usually larger than RA, especially in its proximal segment, but no ultrasound-based studies have reported this finding. The study by Bauman et al. showed no difference between distal RA and distal UA diameters in most of 565 patients [18]. Alternative vascular access sites for left-sided interventions have been a topic of interest, particularly in percutaneous catheter ablations [19-23]. Although the use of this approach could be associated with more vascular spasms, and more challenges could arise because younger populations are affected and larger catheter sizes are used, TRA and TUA could be used more often in clinical settings and elective same-day procedures, such as catheter ablations. In addition, we observed new events of TAO in ultrasonographic examinations during long-term follow-up (after 3 and 6 months). The number of TAO in the study group tripled during the 6-month follow-up. No other studies have shown the percentage of new artery occlusions based on clinical and ultrasonographic longterm follow-up. It is possible that the real number of TAO after using TRA or TUA is higher than documented in trials. The actual number of RA and UA occlusions after 
invasive procedures in long-term follow-up is undetermined and requires further research. Compared with TFA, TRA is associated with a reduction in mortality and MACE, and improve safety, with reductions in major bleeding and vascular complications across the whole spectrum of patients with coronary artery disease [16, 17]. TRA for CAG is now considered as a safe and cost-effective alternative to traditional TFA. In 2016, Kaledin et al. proposed using an alternative vascular access from the distal part of the RA in the anatomical snuffbox (dTRA). In the 656 patients in the dTRA group, radial artery occlusion was observed in $1.5 \%$, hematoma in $0.8 \%$, hand swelling in $0.2 \%$, numbness in $0.6 \%$, ischemic stroke in $0.2 \%$, arteriovenous fistula in $0.2 \%$, pseudoaneurysm in $0.2 \%$ and death in $0.2 \%$ [24]. However, in some cases, TRA or dTRA cannot be used, particularly in those with severe atherosclerosis, TAO of the RA or small diameter of the artery [6]. TUA may be considered for such patient groups. The ultrasonographic measurements in our study also showed that both arteries, RA and UA, had similar diameters. After UA puncture, vessel spasm and an impalpable pulse may result, leading to a longer procedure and more pain. However, there were no statistically significant differences in radiation dose and fluoroscopy time between these two modalities. Based on the analysis of the VAS data, comfort in both groups was similar, regardless of the access. Our study produced several practical results. First, in patients undergoing CAG, TUA is a feasible and safe route for the procedure. Second, the learning curve for this access may influence the initial results and success rates of the approach. Third, the success rate of the operator experienced in TUA was higher than that of the novice TUA operator, and the operator experience was an independent factor for successful TUA. Ultrasonography of the forearm arteries could be a suitable, safe method for selecting the best approach [25].

The study was not appropriate for non-inferiority analysis. The sample size was not calculated due to the limited data of the efficacy of TUA when the study had been designed. Only two operators had specific training in TUA, and this could have affected the observed crossover rate. We did not take into account that the operator experience in TUA may have increased over the course of the study. Analyzing learning curves of less experienced and novice operators could improve our trial. The study was designed with two different strategies of hemostasis and the hemostatic compressions could have been applied under control of blood oximetry. It could have had an impact on reducing the rate of TAOs. The diameter of the RA and UA in the ultrasonographic examination was measured only at one place, which did not reveal the whole size of the artery. Randomization to access site was not based on ultrasonography-derived artery size. Furthermore, the distance from the artery to the bones in the forearm was not evaluated in ultrasonography. Both factors could impact on hemostasis achievement for TUA use. Lost to follow-up patient data ruled out complete estimation of frequency of TAOs.

\section{Conclusions}

TRA was superior to TUA in efficacy. The feasibility and efficacy of TUA depended on the anatomy of the UA (diameter and depth) and operator experience with this access site. TUA was found to be a safe approach for CAG and $\mathrm{PCl}$ and could be used as an alternative method of forearm access. Ultrasonography of the forearm arteries was helpful in selecting the optimal approach and assessing vascular complications.

\section{Perspectives}

No clinical or ultrasonographic risk factors for unsuccessful access attempts, as well as long-term artery patency, have been validated for TUA. Thus, our study attempts to fill this gap in the literature. Moreover, our findings, which show that TUA is a safe approach for CAG and can be used as an alternative method of forearm access, are clinically relevant as operators can use this modality to maintain their skills to achieve a high rate of UA cannulation.

\section{Acknowledgments}

We would like to thank Editage (www.editage.com) for English language editing.

\section{Conflict of interest}

The authors declare no conflict of interest.

\section{References}

1. Agostoni P, Biondi-Zoccai GG, de Benedictis ML, et al. Radial versus femoral approach for percutaneous coronary diagnostic and interventional procedures; systematic overview and meta-analysis of randomized trials. J Am Coll Cardiol 2004; 44: 349-56.

2. Jolly SS, Yusuf S, Cairns J, et al. Radial versus femoral access for coronary angiography and intervention in patients with acute coronary syndromes (RIVAL): a randomised, parallel group, multicentre trial. Lancet 2011; 377: 1409-20.

3. Pristipino C, Trani C, Nazzaro MS, et al. Major improvement of percutaneous cardiovascular procedure outcomes with radial artery catheterisation: results from the PREVAIL study. Heart 2009; 95: 476-82.

4. Valgimigli M, Gagnor A, Calabro P, et al. Radial versus femoral access in patients with acute coronary syndromes undergoing invasive management: a randomised multicentre trial. Lancet 2015; 385: 2465-76.

5. Li YZ, Zhou YJ, Zhao YX, et al. Safety and efficacy of transulnar approach for coronary angiography and intervention. Chin Med J 2010; 123: 1774-9.

6. de Andrade PB, Tebet MA, Nogueira EF, et al. Transulnar approach as an alternative access site for coronary invasive procedures after transradial approach failure. Am Heart J 2012; 164: 462-7.

7. Gokhroo R, Kishor K, Ranwa B, et al. Ulnar Artery interventions non-inferior to radial approach: AJmer ULnar ARtery (AJULAR) 
Intervention Working Group Study Results. I Invasive Cardiol 2016; 28: 1-8.

8. Aptecar E, Pernes JM, Chabane-Chaouch M, et al. Transulnar versus transradial artery approach for coronary angioplasty: the PCVI-CUBA study. Catheter Cardiovasc Interv 2006; 67: 711-20.

9. Hahalis G, Tsigkas G, Xanthopoulou I, et al. Transulnar compared with transradial artery approach as a default strategy for coronary procedures: a randomized trial. The Transulnar or Transradial Instead of Coronary Transfemoral Angiographies Study (the AURA of ARTEMIS Study). Circ Cardiovasc Interv 2013; 6: 252-61.

10. Bertrand OF, De Larochelliere R, Rodes-Cabau J, et al. A randomized study comparing same-day home discharge and abciximab bolus only to overnight hospitalization and abciximab bolus and infusion after transradial coronary stent implantation. Circulation 2006; 114: 2636-43.

11. Lewandowski P, Gralak-Lachowska D, Maciejewski P, et al. Efficacy and safety of kaolin-based hemostatic pad vs. standard mechanical compression following transradial and transulnar access for elective coronary angiography and PCI: RAUL trial substudy. Heart Vessels 2020; 35: 502-8.

12. Dahal K, Rijal J, Lee J, et al. Transulnar versus transradial access for coronary angiography or percutaneous coronary intervention: a meta-analysis of randomized controlled trials. Catheter Cardiovasc Interv 2016; 87: 857-65.

13. Liu J, Fu XH, Xue L, et al. A comparative study of transulnar and transradial artery access for percutaneous coronary intervention in patients with acute coronary syndrome. J Interv Cardiol 2014; 27: 525-30.

14. Geng W, Fu X, Gu X, et al. Safety and feasibility of transulnar versus transradial artery approach for coronary catheterization in non-selective patients. Chin Med J 2014; 127: 1222-8.

15. Fernandez R, Zaky F, Ekmejian A, et al. Safety and efficacy of ulnar artery approach for percutaneous cardiac catheterization: systematic review and meta-analysis. Catheter Cardiovasc Interv 2018; 91: 1273-80.

16. Hildick-Smith DJ, Lowe MD, Walsh JT, et al. Coronary angiography from the radial artery: experience, complications and limitations. Int J Cardiol 1998; 64: 231-9.

17. Ferrante G, Rao SV, Juni P, et al. Radial versus femoral access for coronary interventions across the entire spectrum of patients with coronary artery disease: a meta-analysis of randomized trials. J Am Coll Cardiol Interv 2016; 9: 1419-34.

18. Baumann F, Roberts JS. Real time intraprocedural ultrasound measurements of the radial and ulnar arteries in 565 consecutive patients undergoing cardiac catheterization and/or percutaneous coronary intervention via the wrist: understanding anatomy and anomalies may improve access success. J Interv Cardiol 2015; 28: 574-82.

19. Rillig A, Meyerfeldt U, Birkemeyer R, Jung W. Ablation within the sinus of Valsalva for treatment of supraventricular and ventricular tachycardias: what is known so far? Europace 2009; 11: 1142-50.

20. Tada H, Naito S, Taniguchi K, Nogami A. Concealed left anterior accessory pathways: two approaches for successful ablation. J Cardiovasc Electrophysiol 2003; 14: 204-8.

21. Jiang $\mathrm{H}$, Zhang $\mathrm{M}$, He B, et al. New access for radiofrequency catheter ablation of left-sided atrioventricular accessory pathways: safety and efficacy of the transradial approach. Circ J 2009; 73: 833-7.
22. Lee DW, Kim J, Lee HC, et al. Catheter ablation of a left free-wall accessory pathway via the radial artery approach. Yonsei Med J 2007; 48: 1048-51.

23. He B, Jiang H, Lu Z, et al. Feasibility and safety of transradial approach for catheter ablation of idiopathic left ventricular tachycardia. Clin Res Cardiol 2011; 100: 37-43.

24. Kaledin AL, Kochanov IN, Podmetin PS, et al. Distal radial artery in endovascular interventions. Distal radial artery in endovascular interventions (p.) 2017 Unpublished. https://doi. org/10.13140/rg.2.2.13406.33600.

25. Peruga JP, Peruga JZ, Kasprzak JD, et al. Ultrasound evaluation of forearm arteries in patients undergoing percutaneous coronary intervention via radial artery access: results of one-year follow-up. Kardiol Pol 2015; 73: 502-10. 